\title{
Neuer Paramyxovirustyp als wichtiger Erreger identifiziert
}

\author{
Das neu beschriebene „Humane Meta-Pneumonievirus“ \\ (HMPV) aus der Gruppe der Paramyxoviren erweist sich als \\ relevante Ursache schwerer Atemwegserkrankungen. Kinder \\ mit respiratorischen Grunderkrankungen sind besonders \\ gefährdet. Das zeigt die erste, prospektiv angelegte Studie \\ von K. M. Edwards et al. zur HMPV-Inzidenzraten bei Kindern. \\ N Engl J Med 2013; 368: 633-643
}

In der Literatur wird HMPV erstmals 2001 als mögliche Ursache akuter respiratorischer Erkrankungen beschrieben. Besonders betroffen scheinen Kleinkinder zu sein. Bisher lagen kaum Daten zur Relevanz des HMPV bei kindlichen respiratorischen Erkrankungen vor. Solche Daten sind von großer Relevanz, da das HMPV offenbar besonders Kinder mit entsprechenden Grunderkrankungen (beispielsweise Asthma) gefährdet. Erst epidemiologische Studien machen klar, wie dringend ein Impfstoff benötigt wird.

In der prospektiven Surveillance-Studie erfassten die Autoren in 3 US-amerikanischen Bezirken von 2003-2009 alle behandlungspflichtigen, respiratorischen Neuerkrankungen bei Kindern unter 5 Jahren. Zum Nachweis einer HMPV-Infektion wurde eine serologische Testung

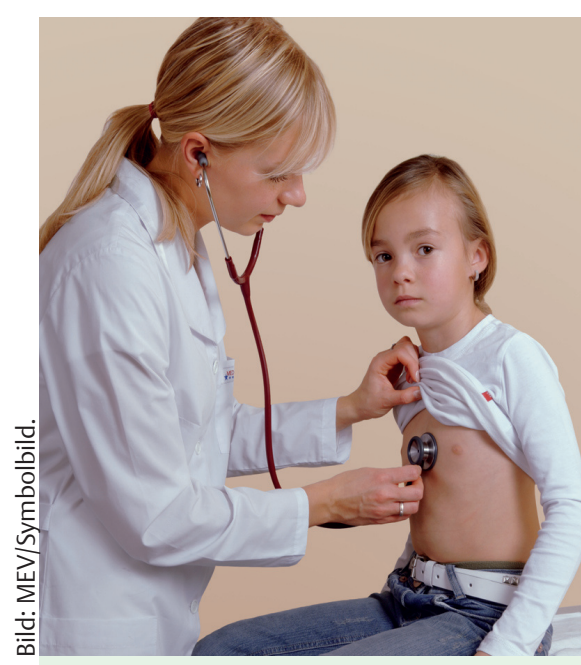

Das 2001 erstmals beschriebene Humane Meta-Pneumonievirus (HMPV) aus der Gruppe der Paramyxoviren gilt als Ursache schwerer kindlicher Atemwegserkrankungen.
(PCR) durchgeführt. Die Forscher differenzierten bei der Auswertung, ob die Kinder ambulant, stationär oder als Notfall behandelt wurden. Respiratorische Vorerkrankungen (z. B. Asthma) wurden ebenfalls erfasst. Nicht in die Studie eingeschlossen wurden Kinder mit anderen schwerwiegenden Grunderkrankungen oder chronischen Symptomverläufen. 770 gesunde Kinder bildeten die Kontrollgruppe. Die Forscher überblickten bei der Auswertung die Daten von 10518 kindlichen Behandlungsfällen. Im ambulanten Bereich fanden sie bei $7 \%$ einen positiven Virusnachweis. Bei den stationär behandelten Kindern lag die Rate bei 6\%. Kinder, die als Notfall in die Ambulanz kamen, waren zu 7\% positiv für HMPV.

In der Kontrollgruppe lag die Inzidenz bei $1 \%$. Die Autoren werten die erhöhten Inzidenzraten als Beleg dafür, dass das HMPV eine relevante, bisher nicht berücksichtigte Ursache akuter respiratorischer Erkrankungen bei Kindern darstellt. Die Detailanalyse zeigte eine besondere Gefährdung von Kindern mit respiratorischen Grunderkrankungen: Hier war der Virus in 40\% der stationären Fälle nachweisbar.

\section{Fazit}

Die HMPV-Infektion wird nach Angaben der Autoren als Ursache akuter respiratorischer Erkrankungen bei Kindern bisher zu wenig beachtet. Die Inzidenzraten erreichen Dimensionen, die als typisch für RSV und Influenza gelten. Besonders Kinder mit einer respiratorischen Gefährdung werden von der Entwicklung eines HMPV-Impfstoffs profitieren.

Dr. Horst Gross, Berlin

\section{Test sagt lebenslange Erkrankung vorher}

Die Erforschung der genetischen Risiken von Asthma könnten zur Entwicklung eines Tests führen, der vorhersagen kann, welche Kinder ihr Leben lang betroffen sein werden und welche nicht. US-amerikanische Wissenschaftler der Duke University haben herausgefunden, dass Kinder mit einem höheren genetischen Risiko ein um 36\% höheres Risiko haben, an einer ernsten und andauernden Erkrankung zu leiden. Laut dem Team um Daniel Belsky ist es derzeit allerdings noch zu früh, an einen zuverlässigen Test zu denken. Details der Studie wurden Ende Juni in der Fachzeitschrift Lancet online veröffentlicht. Die Studienergebnisse sollen helfen, jene Menschen zu identifizieren, deren Erkrankung einen schweren Verlauf nehmen könnte.

Die Erhebung hat 15 verschiedene Bereiche des menschlichen Genoms identifiziert, die mit Asthma in Zusammenhang stehen. Diese Daten, in Kombination mit einer großen Studie aus Neuseeland, für die mehr als 1000 Personen seit ihrer Geburt begleitet wurden, ermöglichten es, das genetische Risiko einer Erkrankung für 880 Menschen zu berechnen. In einem nächsten Schritt wurden die Entwicklung und das Fortschreiten der Krankheit von der frühen Kindheit bis fast zum 40. Geburtstag analysiert. Kinder mit einem höheren genetischen Risiko litten eher an schwerem Asthma, das sie auch als Erwachsene noch hatten. Sie zeigten auch häufiger Probleme mit der Lungenfunktion, fehlten eher in der Schule oder am Arbeitsplatz und mussten häufiger wegen ihres Asthmas ins Krankenhaus.

Werden weitere Risikogene identifiziert, sollen sich auch die Vorhersagemöglichkeiten verbessern. Es wird, nach Meinung der Wissenschaftler, allerdings noch lange dauern, bis genetische Risiken routinemäßig für die Arbeit in der Praxis eingesetzt werden können. Die aktuelle Studie trägt jedoch zu einem besseren Verständnis dieser Krankheit bei, wodurch sich auch die Behandlungsmöglichkeiten verbessern.

Nach einer Mitteilung der pressetext Nachrichtenagentur GmbH 